\title{
Parent and child perspectives on the nature of anxiety in children and young people with Autism Spectrum Disorders: a focus group study
}

Article

Accepted Version

Ozsivadjian, A., Knott, F. and Magiati, I. (2012) Parent and child perspectives on the nature of anxiety in children and young people with Autism Spectrum Disorders: a focus group study. Autism, 16 (2). pp. 107-121. ISSN 1362-3613 doi: https://doi.org/10.1177/1362361311431703 Available at https://centaur.reading.ac.uk/26434/

It is advisable to refer to the publisher's version if you intend to cite from the work. See Guidance on citing.

To link to this article DOI: http://dx.doi.org/10.1177/1362361311431703

Publisher: Sage Publications

All outputs in CentAUR are protected by Intellectual Property Rights law, including copyright law. Copyright and IPR is retained by the creators or other copyright holders. Terms and conditions for use of this material are defined in the End User Agreement. 


\section{CentAUR}

Central Archive at the University of Reading

Reading's research outputs online 


\title{
Parent and child perspectives on the nature of anxiety in children and young people with Autism Spectrum Disorders: a focus group study
}

\begin{abstract}
Anxiety disorders are common among children and young people with Autism Spectrum Disorders (ASD). Despite growing knowledge about the prevalence, phenomenology and treatment of anxiety disorders, relatively little is understood about the nature and impact of anxiety in this group and little is known about autism-specific factors that may play a role in the increased prevalence of anxiety disorders. In this exploratory study, we report on a series of 5 focus groups with 17 parents of children and adolescents with ASD and anxiety. Across groups, parents gave strikingly similar descriptions of the triggers and behavioural signs associated with anxiety. Another consistent finding was that many parents reported that their children had great difficulty expressing their worries verbally and most showed their anxiety through changes in their behaviour. The impact of anxiety was reported to often be more substantial than the impact of ASD itself. The implications of the focus group findings are discussed in relation to existing literature.
\end{abstract}




\section{Introduction}

Autism Spectrum Disorders (ASD) are lifelong conditions with varying, but often high, levels of associated cognitive, communication, social, behavioural and mental health impairments (APA, 2000). Anxiety in particular is now a well-recognised problem for children and adolescents with ASD (see MacNeil, et al., 2009; White, et al., 2009 for reviews). Furthermore, reported rates of anxiety are higher than those in children with language disorders (Gillott et al, 2001), conduct disorder (Green et al., 2000), or in clinically anxious typically developing children (Farrugia and Hudson, 2006). Knowledge about the prevalence, phenomenology and treatment of anxiety in ASD is increasing, but several issues remain unexplored regarding how anxiety is manifested in children and adolescents with ASD. This has implications both for the way anxiety is assessed and for the development and implementation of effective interventions for this vulnerable population.

Phenomenology of anxiety disorders in childhood

Epidemiological studies show that typically developing children and adolescents commonly experience anxiety disorders (Muris and Broeren, 2009), and these may be the most common psychological disorder of childhood (Cartwright-Hatton et al., 2006). Anxiety disorders in childhood frequently persist into adolescence and adulthood, often occur with other disorders and can have a negative impact on functioning in areas such as academic and social performance (Rapee, et al, 2009). 
Anxiety itself may be best conceptualised on a continuum (Schniering, et al 2000), with many children experiencing transient worry, stress or anxiety. At times anxiety can enhance performance or offer protection from threat, and furthermore, at some developmental stages, certain types of anxiety are so common that they may be seen as normative; for instance, separation anxiety around the ages of 12-18 months. However, anxiety disorders can be differentiated from 'normal' fears and worry on the basis of severity of symptoms, persistence and impact on functioning (Beesdo, et al., 2009). Current estimates suggest that prevalence of all anxiety disorders is between 2.5 and 5\%, affecting twice as many girls as boys (Rapee et al., 2009) with cumulative prevalence rates indicating that $9.9 \%$ of children and adolescents under 16 are likely to experience an anxiety disorder (Costello, et al 2003).

Using a cognitive-behavioural approach to anxiety in childhood, the clinical features of anxiety may be conceptualised as physiological, cognitive, emotional, and behavioural. Physiological changes include somatic complaints such as nausea, headaches, sweating and muscle tension. The emotional component encompasses feelings of fear, worry, dread or irritability. Cognitive symptoms often reflect catastrophic predictions and expectations of failure to cope. Finally, the predominant behavioural symptom of anxiety is avoidance, though hypervigilance and checking are also common. 
There are now several well conducted prevalence studies showing that anxiety occurs in children with ASD at a higher rate than in the typically developing population. Although prevalence rates vary from 11 to $84 \%$ (White et al, 2009), most studies indicate that around half of children with ASD meet criteria for at least one anxiety disorder (e.g. Simonoff, et al., 2008). Knowledge is also emerging of the relationship between anxiety and ASDspecific factors, including impoverished empathy and social skills (Bellini, 2004), theory of mind and central coherence difficulties (Burnette, et al., 2005) and sensory over-sensitivity and responsivity (Green and Ben-Sasson, 2010). However, studies examining the phenomenology of anxiety in ASD have generally employed measures that were standardised with typically developing children. They may not, therefore, adequately reflect the specific ways in which anxiety may manifest itself in children with ASD. Furthermore, the way anxious symptoms in ASD are measured and described varies significantly between studies (White et al., 2009), further limiting our understanding of anxiety in this group. Anecdotally, many clinicians recognise that the triggers and presentation of anxiety in children with ASD can be markedly different to the typically developing population and may not be captured by existing standardised measures. For example, anxieties associated with changes in routine do not fit easily into any particular category using current diagnostic criteria. There can also be difficulties differentiating between anxiety symptoms and other problems relating to regulation of emotion, particularly anger or low mood; or, indeed, autism symptoms themselves (Muris et al., 1998). 
Despite emerging knowledge, little is known about the atypical way that anxiety may present in children and young people with ASD. The difference in presentation of depression between ASD and non-ASD populations has been discussed (e.g. infrequent reporting of typical depression symptoms such as feelings of worthlessness and guilt, increased aggressive behaviour or selfinjurious behaviour; Stewart et al; 2006). To date, a similar investigation on the nature and presentation of anxiety in ASD has not been published, to the authors' knowledge.

The above limitations notwithstanding, emerging literature exists about the use of cognitive behaviour therapy (CBT), the treatment of choice for typically developing anxious young people, with individuals with ASD (Lang et al., 2010; Moree and Davis, 2010). Recent randomised controlled trials suggest that enhanced and adapted CBT can successfully treat anxiety disorders in high functioning children with ASD (e.g. Wood et al., 2009), though currently it is not clear which adaptations are crucial or which modifications are required for which anxiety disorder (Moree and Davis, 2010). Several issues limit the development of effective interventions. One problem relates to the lack of appropriate measures developed and standardised for children with ASD, which impacts on the assessment and interpretation of change and outcome in efficacy studies. Additionally, the lack of available information about the atypical presentation of anxiety in ASD renders it difficult to implement the AACAP Practice Parameter recommendation (Connolly and Bernstein, 2007) that anxiety treatments are matched to child and family characteristics. Understanding more about the factors triggering and maintaining anxiety in 
ASD, and how children with ASD and their families experience, think and talk about anxiety, could inform the development and implementation of autismspecific interventions for anxiety for this population.

Therefore, despite many promising advances in the literature, our understanding of anxiety in children with ASD remains relatively limited. Given the limitations of quantitative methodologies using standardised measures developed for typically developing children largely employed so far in anxiety research in ASD, the present study uses a qualitative, "bottom-up" approach to furthering our understanding of anxiety in this population. We report on data obtained from focus groups with parents and children with ASD as a first step in identifying the triggers, behavioural signs and cognitive processes associated with anxiety in this group through parental perspectives, with the aim of increasing our understanding of the presentation of anxiety in children with ASD.

\section{Method}

\section{Participants}

Participants were recruited via the National Autistic Society and parent support groups in Berkshire, London and Surrey, via email newsletters sent to all members of those groups. An invitation letter accompanied the information sheets, inviting parents to attend a focus group specifically discussing anxiety in ASD in order to find out more about the causes and processes of anxiety in this group. Parents of children with ASD and any experience of worry or anxiety aged 7-18 years were invited to participate. No incentives were 
provided for participating. No exclusion criteria were set with regards to children's functioning, comorbid diagnoses or whether they had received treatment for anxiety in the past, as this was an exploratory study and obtaining rich and varied perspectives on the experience of anxiety was prioritized.

Five parent groups in four localities were conducted in total. Altogether, 17 mothers attended, who were parents of 19 children with a diagnosis of ASD; 7 girls (age range $7-18$, mean age: 11 ) and 12 boys (age range 7-16, mean age: 12). Children had received ASD diagnoses from local clinical services: 5 had a diagnosis of Asperger Syndrome, 6 had a diagnosis of high functioning autism, 1 had a diagnosis of atypical autism and 7 had a diagnosis of ASD or autism. All parents reported that their children experienced significant levels of anxiety which impacted on their functioning. Six had received cognitive behavioural or behavioural treatment for anxiety at some stage.

All the children of participating parents were invited to participate in children's focus groups; however, few accepted. Four boys aged 10-12 years with Asperger's syndrome and high-functioning autism accepted, and one focus group was held in order to explore the children's own views. Findings from the children's focus group are reported in less detail due to the small numbers recruited.

\section{Topic guide}

The topic guide took into account the literature relating to the prevalence, phenomenology and treatment of anxiety in ASD, cognitive behavioural 
models and treatment of anxiety in typically developing children and the clinical experience of the professionals leading the groups (Appendix 1). Prior to the research groups, two pilot groups were held with parents from a local parent support group to ensure topics were comprehensive and relevant and to develop appropriate questions for use in the main groups. Once finalised, the same topic guide, adapted to be age-appropriate, was used for the children's group.

\section{Procedure}

Mothers met in groups of 2-4 for about 2 hours. Groups were facilitated by two of the authors (AO and FK), chartered clinical psychologists experienced in working with children and adolescents with ASD. The facilitators outlined the purpose of the group and asked mothers to introduce themselves and talk a little about their children, before using the topic guide to generate discussion. Care was taken not to ask leading questions, and to let discussions develop freely with as little interference from the facilitators as possible, beyond ensuring that all areas were covered. However, at times it was necessary to clarify issues (e.g. when parents were describing angry/oppositional behaviour rather than anxious behaviour).It was acknowledged that it was not always easy to distinguish between the two; the facilitators regularly clarified this by defining anxiety in lay terms as particularly pertaining to worry or stress, rather than aversion or oppositionality. For example, when parents mentioned repetitive behaviours, facilitators clarified whether this worsened at times of anxiety. Facilitators also ensured that the discussions were not monopolized by one member of the group only and made efforts to give all 
members opportunities to contribute and express their views and experiences. The children's group met at the same time as the parents' and followed a similar topic guide and procedure, albeit with more breaks for snacks and games. The sessions were audio -recorded using digital voice recorders and later transcribed verbatim.

\section{Ethical Considerations}

Ethical approval was obtained from King's College London and the University of Reading Research Ethics Committees. The groups had the potential to raise upsetting memories for participants. The facilitators ensured that boundaries for 'safe' discussion and confidentiality were set and maintained throughout the sessions. Information was given to parents about where to seek further support if required.

\section{Data analysis}

Following focus group methodology in analyses (Kitzinger, 1995; Krueger and Casey, 2000), transcripts were analysed using thematic analysis (Aronson, 1994). They were initially scrutinised to identify data that related to predefined categories (triggers, behavioural, somatic and cognitive signs). Relevant data from all transcripts were then read and re-read to identify patterns and subthemes within each predefined category, using an iterative process resulting in the most parsimonious number of sub-themes, yet ensuring all material could be sub-classified. Once the coding scheme had been established, one complete transcript was double-coded by both researchers to ensure consistency of approach. In accordance with the focus group methodological 
principle of "words not numbers" (Grudens-Schuck et al., 2004; Fern, 2001; Kitzinger, 1995), transformations of qualitative information into quantitative data were not carried out, as in a group discussion, there is difficulty recording every manner of interaction, such as nodding or other communications of agreement or disagreement.

\section{$\underline{\text { Results }}$}

The focus groups generated rich information and a number of themes relating to anxiety emerged that were highly consistent across groups. Originally, 'worries about school' was included as a separate category, but it became apparent that anxieties about school were so common that they pervaded all the categories. Therefore, school-related anxieties were included in the category to which they pertained, such as worries about other pupils (social), school work (performance expectations) or noise levels (sensory).

\section{Parents' groups}

\section{Anxiety triggers}

Several key themes emerged within this category. These were classified as triggers relating to change or routines; social or language-related triggers; specific fears or phobias; sensory triggers; triggers relating to obsessions; and triggers relating to high expectations in performance or organization. By far, the most common triggers for anxiety related to change and social situations.

Change or disruption to routine. Examples included getting changed for $\mathrm{PE}$, having a supply teacher, a delay in a flight or being stuck in traffic, and changing schools. "At the beginning of term they drop the first period of the 
first day. M couldn't sleep that weekend, he has to bring the books in for that period even though he doesn't have it". Non-routine events such as holidays or sports days also featured frequently. Making choices was also anxietyprovoking, often because of a fear of making the wrong choice. "Choices that seem totally trivial, they get escalated in their heads". Similarly, situations usually considered pleasurable, such as Christmas or birthdays, were often anxiety-provoking because of the element of change and unpredictability. "He can't cope with the surprise of it (Christmas). It's the not knowing, the routine thing. His Dad had a complete meltdown when he found out what I had done [shown child his presents in advance]... He said 'it's meant to be a surprise, he's not going to enjoy it'...No, he's not enjoying it now because he doesn't know".

One parent summarised vividly why change is so aversive to children with ASD: “There's a visual [memory] bank already there...you don't have to update it all the time [if things stay the same]. 'I've seen this before, I've scanned it', I'm not there thinking 'oh my God, I have to log all of this, I can't do this because it makes my head hurt. And also when I'm logging it, l've got to look out for lions and tigers because they might kill me'. Their heads are full of junk and they cannot process it...I think a lot of it is the basic desire to survive."

Social/ language triggers. Common social worries such as being the centre of attention were mentioned here, as well as fear of being ridiculed. For example, one mother commented: "He'll make some funny quip which is totally appropriate and everything else, then on the way home he'll say 'was 
that funny? Did that go down well?' And I'll say 'Yeah, it was ok' and he'll say 'Cos I don't want to let you down...you know, by not being funny'. And I think, it must be such a worry, when you're constantly worrying about what you're saying, how you're saying it, who you're saying it to...it must be really hard". More ASD-specific social worries included Theory of Mind related problems, e.g.: "He likes rules...it's those situations where there aren't any rules and you're expected to behave in a certain way (that cause anxiety), he doesn't know...". Self-awareness of social difficulties in itself caused anxiety, e.g.; "She's in the playground, and completely on her own. I think nowadays she notices and there's a growing amount of anxiety over that".

Specific fears and phobias were also mentioned. Some were common fears, such as spiders, lifts and injections. Others were more idiosyncratic: one boy had a fear of chocolate coming out of his belly-button after watching the film 'Charlie and the Chocolate Factory'.

Sensory triggers were often cited, e.g. loud noises, too many voices, certain smells. "H becomes extremely anxious when she hears a baby cry...she's got a radar from 700 miles..."; "if he was to walk into a noisy room, he'll get really stressed". "If she moved the cucumber near $H$, he's heaving, panicking".

For another child, a fear of the smell of school (and hence fear of contamination of objects with this smell) related to his OCD rituals.

Routines and Obsessions. Less frequently, but consistently mentioned, were triggers relating to obsessions, in particular being prevented from 
carrying out certain activities. "He carries the Argos catalogue around with him and gets upset if he can't have it".

Anxiety triggered by too many demands or expectations. Lastly, there was a group of comments which we termed demands and expectations, referring to overwhelming expectations placed on the child by themselves or, most frequently, by others (such as expectations of independence, school achievement or social conformity). For example: "From other people's perspective, he's being naughty...the reason he's been sick is that they had a rota for the children to sweep the floor, the minute he was on the rota to do that, he was sick [with worry]"; "As J's getting older, there are expectations that when you are in year $x$, you should do this and you should do that, like carry messages home: no, she can't. She goes to school in uniform and they'll all be in home clothes: 'oh, didn't she tell you?' No, she didn't"; "I think it's something they perceive as a demand, and the feeling they are going to be asked something they can't produce".

\section{Presentation of anxiety}

\section{Behavioural indicators}

Within this category, five main themes emerged.

Challenging behaviour. The majority of comments in all groups fell into this and the next category. 'Meltdowns' and verbally and physically aggressive behaviour at times of anxiety were frequently mentioned. "She gets very agitated, she wrings her hands and starts to shout. In the past she was violent, she's hit us, bitten me..."; "He's swearing a lot more now, the anxiety 
is coming up in swearing as he's finding a way of expressing it rather than beating himself up". Self-harm was also mentioned by several parents: "He's learnt, if I harm myself, he's instantly taken away". Children's well-being was also compromised in other ways: "Safety awareness goes down - he's flailing around at anxious times, destroys things, rips things off the walls".

Avoidance/withdrawal/escape. Many comments pertained to avoidance or withdrawal at times of anxiety: "He becomes unreachable - you can just feel he's close to boiling point"; "When she's anxious, she tries to close herself off and roll into a ball, you know, the smallest I can become"; "At school last year, I didn't realise he was spending most of his time actually hiding under the table or behind the trolley. It was literally the foetal position, when he gets anxious, that he's going into"; "The extreme times (of anxiety), I can't even get her to post a letter with me at the end of the road - 'I'm going nowhere".

Arousal. Many parents noted that the first signs of their child becoming anxious included an increase in activity levels or nervous behaviour. For example: "If you didn't know him, you might think he has hyperactivity because he goes completely 'woohoo'; "We have an expression in our house - 'he's a little skippy today"'. "With J she becomes edgy and gets what I call flicky eyes. Her eyes are just not still, she's checking where everybody is, what they're doing".

Sensory behaviour. Some parents commented on an increase in sensory behaviour at times of anxiety, e.g. nail-biting, humming and shouting, chewing of clothes. "Occasionally, he will do this with his hands if he's feeling stressed, 
sort of twist things". "Even now, at 16, when he's in a high state of anxiety... he'll go down there and jump about and make loads of baby noises".

Obsessional and repetitive behaviours. Many parents reported an increase in sensory, obsessional and repetitive/stereotyped behaviours at times of anxiety: "Depending on how anxious he is, all the trains have to go into their station: it eats up time and gets you so frustrated"; "He homes in on a subject and it becomes so intense"; "J will repeat questions, the same question over and over again until she's satisfied with the answer".

\section{Somatic indicators}

Parents occasionally reported somatic or physiological changes at times of anxiety. Disruption to sleep was most commonly cited, followed by an increased frequency of stomach aches or nausea. "When he was young, he said it felt funny. That was the beginning and now it's an 'I'm going to be sick' feeling". And at the more extreme end: "With $H$ it [anxiety] comes out in physical illness, boils, red bumps, signs on the skin". "He goes white, clammy, black eyes, classic 'I'm anxious' physiological signs that he's anxious".

\section{Cognitive indicators}

Parents were asked whether their child reported any anxious thoughts. An overwhelming theme was the difficulty that children had in telling others when or why they were anxious, and the "detective" process that many parents had to go through to access their children's thoughts and experiences. In relation to cognitive indicators, two main themes emerged: cognitive processes and content of cognitions. 
Cognitive processes. Comments relating to the difficulty children had in sharing their thoughts were frequent and arose consistently in every group. "It's really hard because they haven't got the verbal skills to tell you and by the time they tell you it's too late"; "And the problem is, he's got it all in his head and he can't tell me what's going on"; "It's not out of the blue, it's something he's been thinking about and processing but it could be two days old." "Normally about a 2-3 week delay between something happening and me being able to tell and by the end it's so embedded you've got to disentangle the whole thing before you get to the point"; "It just occurred to me, she never says 'I think' or 'I feel...', she says 'my head is so full of worries I can't think",:

There were also comments pertaining to the style of thinking that led to or exacerbated anxiety. In line with cognitive behavioural theories of anxiety (e.g. Beck and Clark, 1997) some parents described typical cognitive distortions or 'thinking errors': "She gets into a situation where she imagines what they're thinking about her, she doesn't actually understand what's going on"; "Thought processes are always negative - she remembers negative events in great detail...". Another parent described a processing style akin to faulty memory processing described in post traumatic stress models (Ehlers and Clark, 2000): "It's still in the memory bank and will still trigger anxiety, because it hasn't been filed away. Nobody's the filer, the filing doesn't work, it's never ever put away"; "Putting it in some sort of order before they can talk about how they felt...for $M$ that order will be really jumbled for a while, so he'll tell you bits of it, he might even tell you key words and in between those key words he's on the floor and crying". 
Cognitive content. Some specific worries that the children had were mentioned, such as: "I'm worried I won't get the grades I want"; "They're laughing at me". Most of the cognitions parents mentioned reflected depressive or mixed anxious and depressive thinking and common cognitive errors of predicting the future, all-or-nothing thinking and catastrophising: "People don't like me"; "I am totally useless" ; "The whole world's against me"; "I ruin everything"; "Childhood was hard but adulthood will be even harder".

\section{Perceived differences between anxiety in children with ASD and typically developing children}

As many parents also had children without ASD, we also enquired whether they perceived any differences between anxiety in their children with and without ASD. Despite some potential limitations of asking parents this question (i.e. they may have limited experiences of typically developing children and/ or their other children may be at higher risk for a diagnosis of ASD or ASD broader phenotype), we were nevertheless interested in finding out what parents perceived as differences, and indeed clear themes emerged. With regards to presentation of anxiety, the behavioural rather than verbal expression of anxiety, and the time delay between an event and expression of anxiety were consistently perceived by the parents in all groups as key differences. Parents reported that anxiety in their children with ASD was typically prolonged and more intense and that their children were more difficult to reassure or soothe. Some parents described their children with ASD as being constantly on the edge of anxiety, or permanently in "fight or flight" mode: "He's never actually happy -it's [anxiety] his default setting". Another 
theme was that many things usually considered pleasurable (e.g. Christmas) are actually anxiety-provoking for children with ASD due to their difficulties in coping with change, or dislike of surprises.

In terms of triggers, social worries and anxiety in relation to change were identified as different in their children with ASD compared to their siblings or other children without ASD: "Social anxiety is inherent, which leads to awkward social situations"; "The difficulties with change are different".

\section{Impact of anxiety}

The impact of anxiety on families and the children themselves became particularly apparent when behavioural indicators of anxiety were discussed. Indeed, some parents expressed a view that anxiety often had a more of a negative impact than the autism itself.

Impact on parents. The majority of comments fell into this category. Parents expressed feelings of guilt, such as not being able to help their child effectively with their anxieties and not always being able to contain their own frustrations: "It's really difficult, you want to try and understand what's happened but there's no way you can go back to it". Feelings of sadness were also described: "It's really sad isn't it, I think she feels like every day is a mountain to climb [because of the anxiety] and it's overwhelming".

Parents also described increases in their own anxiety: "I have noticed that his anxiety 'transfers' onto us. We frequently wake up with J things on our minds". The impact of public perceptions was also discussed, particularly in relation to challenging behaviours as a way to express anxiety: "And normally you get 
looked at as if you're the worst parent in the world"; "'People probably think I'm publicly disembowelling him".

Impact on the children. "If you think about it, [it's like] having exams every day of their lives and that can't be nice for anybody, to live under that amount of stress all the time"; "As a person, we lost him through the anxiety".

Impact on siblings. Social and emotional implications for siblings were described: "We limit who [sibling] can have round because he'd [child with ASD] panic"; "She [sibling] needs support as well because sometimes it drives her mad"; "She worries she will be attacked".

\section{Children's group}

Much of what the 4 boys said in their focus group mirrored the mothers' comments. When asked 'what makes you anxious?' many comments pertained to change in routine, sensory triggers (such as teachers shouting), and social worries (such as being at a friend's house without their parents and not knowing what to say). Worries around being bullied also featured frequently.

The children were able to identify behavioural signs of anxiety, reporting that they experienced somatic symptoms such as a racing heart, out of control breathing, butterflies, wobbly legs, feelings of nausea and sweating. They also identified some thought processes such as "thoughts racing out of control" and "my mind blacking out"; one child referred to "the voices in my head". Identified cognitions included: "I'm going to get hurt"; "They might insult me"; and "Will they like me?" However, few clearly articulated cognitions were 
identified and, as a rule, boys had considerable difficulty explaining why certain situations triggered anxiety: "Just felt weird and strange, it's unlogical"; "It was change, it just won't be the same".

\section{Discussion}

Using focus group methodology, this study yielded rich, clinically useful and very consistent descriptions of the triggers and manifestations of anxiety in children and adolescents with ASD. The parents' perspectives on the anxieties of their children with ASD demonstrate that there are many overlaps with anxiety as experienced in the general population. Shared triggers included social worries, specific phobic stimuli, and expectations that are, or are perceived to be, too demanding. Common features of the presentation of anxiety include an increase in arousal and avoidance/escape behaviours. The information gained on cognitions, although limited, also indicates some overlap in the cognitive processes of anxiety in the ASD and typically developing populations.

However, a number of autism-specific triggers and symptoms of anxiety were identified. In their children with ASD, key features were the intensity, pervasiveness and persistence of the anxiety. Changes and disruptions to routines, sensory sensitivities and social difficulties rooted in difficulties with perspective-taking and social expectations appeared to be ASD-specific triggers. Situations that were reported to trigger anxiety often reflected autismrelated difficulties in processing style or sensory sensitivity. The majority of comments about the presentation of anxiety fell into the categories of challenging behaviour and avoidance/withdrawal, indicating that behavioural 
means are the main ways of expressing anxiety in children with ASD. When asked about their anxious thoughts, the most prominent theme that emerged was the difficulty that children with ASD have in expressing their anxiety verbally, particularly at times of acute distress. Although this is also true for many typically developing children, it may be a particular difficulty for children with ASD given the challenges they face in understanding and expressing emotions and pragmatic language difficulties. The mixed anxious/depressive nature of many of the cognitions reported indicates the high comorbidity of anxiety and low mood in this group. Many negative cognitions may be a reflection of the reality of the situations they have to deal with, as Jahoda et al. (2009) note with regard to a population with intellectual disability.

The impact of anxiety was evident in all groups. Children with ASD were described as being highly dependent on their parents in managing their anxiety, and this presented significant challenges to families. Parents provided moving accounts of the toll their children's anxieties can take on their own emotional wellbeing, that of their other children, and family life in general. Many emphatically agreed that anxiety frequently had a greater impact on family functioning and flexibility than ASD itself, further reducing children's and families' quality of life.

\section{Limitations}

The findings presented here need to be interpreted in light of the study's limitations. One important limitation is the small sample size and representativeness of the participants; who were a self-selected group from a relatively small geographical area. The lack of an incentive may have resulted 
in a particularly motivated group. Another limitation was the lack of detailed information about demographics, language skills, cognitive functioning or adaptive skills of the children involved, which was beyond the scope of this exploratory study. However, it is likely that the findings of this study can be tentatively generalized to children in the higher functioning end of the ASD spectrum. A general limitation of focus group methodology is that discussion can be dominated or sidetracked by individuals, or may not be representative of other groups (Kitzinger, 1995; Krueger and Casey, 2000), and therefore we have made an effort to present and focus on themes identified across all the focus groups. At the same time, the interaction of group members is likely to result in richer conversations and wider range of themes discussed (GrudensSchuck et al., 2004). Another limitation was the challenge of ensuring that parents were at all times referring to anxiety and not non-specific negative affect, such as aversion or discomfort. The facilitators made efforts to ensure that all comments included in this paper specifically pertain to anxiety, but recognise that, due to the limited ability of their children to express their feelings, parents may at times have been making assumptions about anxiety triggers or anxious behaviours. Thus, although we made every effort to clarify parents' comments by reiterating definitions of anxiety when necessary, we are aware that parent examples may at times have represented non-anxiety specific symptoms and behaviours.

Finally, the number of young people with ASD who participated was too small to further explore similarities or differences between parent and child reports 
of anxiety or differences in anxiety as a function of child characteristics with any confidence.

\section{Theoretical and Clinical implications of the focus group findings}

The present findings provide further insight into the appropriateness of using standardised measures consisting of pre-defined symptoms or manifestations of anxiety, developed for the general population, with children with ASD. Although existing measures are useful for comparison purposes, our study shows that when parents and children with ASD are invited to describe their experiences in a semi-structured, non-directed manner, they largely focus on features more specific to the ASD population that are not typically measured on most anxiety measures. Although measures exist that do evaluate broad emotional difficulties in children with developmental disabilities (e.g., the Developmental Behaviour Checklist, DBC; Einfeld and Tonge, 1992), a significant gap remains in the resources available to clinicians and researchers for measuring ASD-specific aspects of anxiety, now well demonstrated to be an extremely common presenting clinical problem in ASD. There are also implications for the inclusion of observational methods in the assessment of anxiety: using multiple informants, multimodal assessment techniques and methods has been recommended (MacNeil et al., 2009). These findings may also be useful for clinicians working with children and young people with ASD, particularly those attempting to engage children with ASD in CBT. Although there is now good evidence that, with adaptations, CBT can be an effective means of treating anxiety in young people with ASD (e.g. Chalfant et al., 2006), clinicians may need to consider whether CBT is 
the most appropriate intervention, or whether other approaches such as behavioural work through parents or environmental modifications may be more appropriate, as even for verbally-able children, accessing or modifying cognitions may prove too challenging. However, clearly CBT is an effective intervention for many children with ASD, and the involvement of parents can further improve outcomes (Moree and Davis, 2010). Our findings support the view that adaptations to $C B T$, such as adding ASD-specific components to the treatment protocol, are important, relevant and acceptable to parents of children with ASD (Sze and Wood, 2008).

Attempts at developing a theoretical model of anxiety in individuals with ASD are still very much at the stage of theorizing, but two have been proposed to date. Gaus (2007) proposed a model developed for the adult population which includes vulnerability to depression as well as anxiety, and highlights social cognition deficits, information processing difficulties, sensory dysfunction and increased risk for negative life events (i.e. rejection and bullying) leading to anxiety and depression. Wood and Gadow (2010) more recently proposed a theoretical model of clinical anxiety in ASD which included ASD-related stressors (social confusion, peer rejection, prevention of repetitive behaviours and aversive sensory experiences) leading to two potential mood dysregulation pathways (social anxiety and other anxiety or depression types) which in turn result in increased social avoidance, increased severity of ASD behaviours, increased behavioural difficulties and reduced quality of life. The themes identified by the parents in our study (see Figure 1) very closely map onto the factors put forward by Wood and Gadow (2010), and provide some 
initial support for the face and external validity of this proposed model and its applicability to the real-life experiences of anxious young people with ASD and their families. Wood and Gadow also highlight the importance of integrating these factors in any attempt to systematically understand how anxiety is developed and maintained in this population.

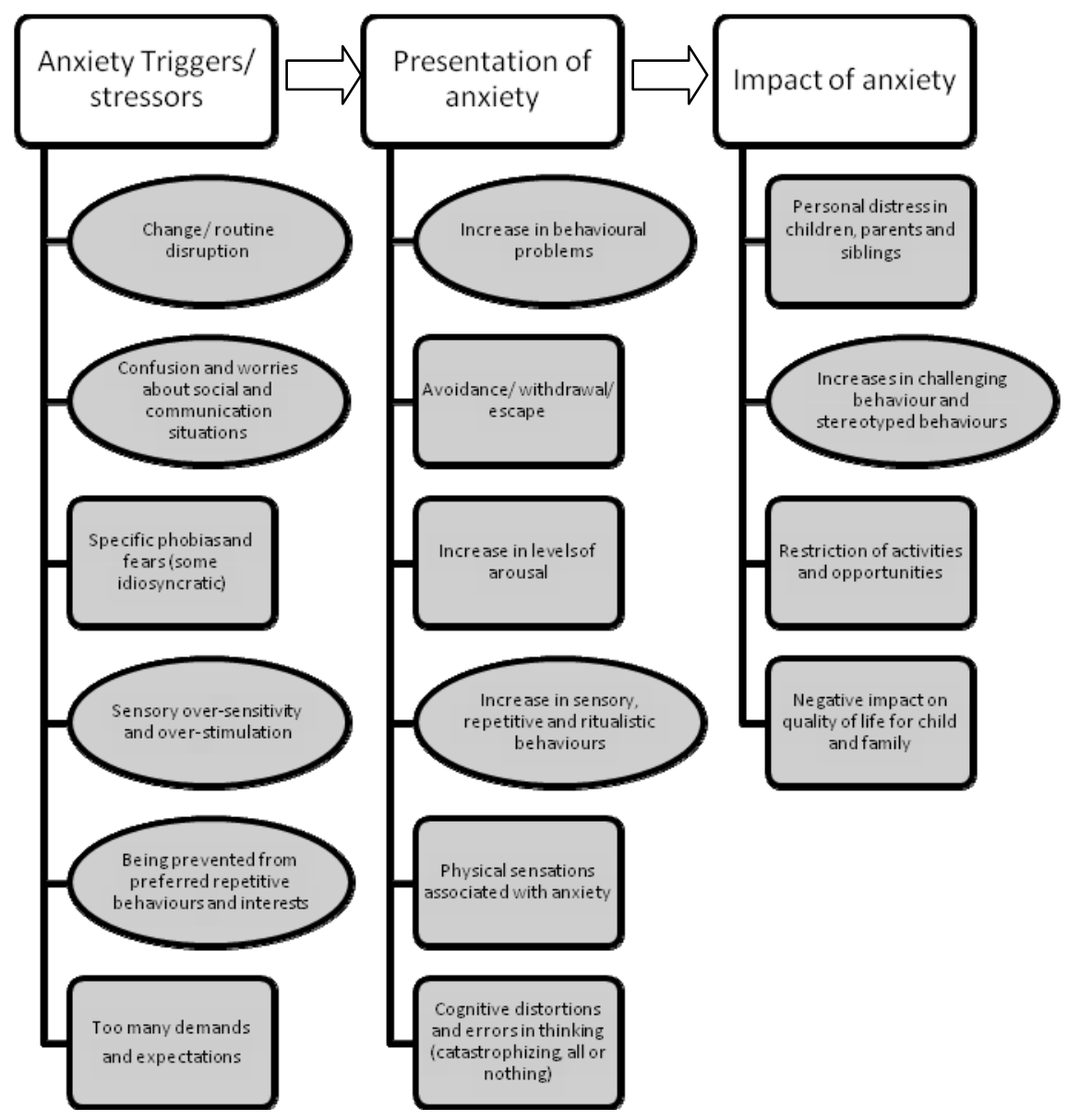

Figure 1. A summary of key themes identified by parents in relation to triggers, symptoms and impact of anxiety in their children with ASD mapped in relation to the Wood and Gadow (2010) hypothetical model 
of anxiety in ASD (potentially ASD-specific themes are identified in oval shapes)

\section{Conclusions}

Despite the limitations, our findings have taken us some way towards our aim of furthering our understanding of the presentation of anxiety in this population. We were struck by the consistency of responses across groups, with parents identifying very similar triggers, predisposing and maintaining anxiety factors. However, questions about causal relationships and pathways leading to increased prevalence of anxiety in this group remain unexplored. Future research could focus on larger groups, including control samples, systematically investigating the link between ASD cognitive processing styles and anxiety. For example, do children with ASD have a negative processing bias towards threat-related information? Do they demonstrate encoding or retrieval memory problems that lead to poor autobiographical memory and /or a sense of threat? In terms of intervention, how can we assist children to access their cognitions, both in therapy and real life situations, or indeed, are the cognitive elements of interventions for this group effective, and if so, for whom? Further exploring the similarities and differences between shared and ASD-specific triggers, manifestations and effects of anxiety using a variety of methodologies will contribute to the development of more appropriate assessment measures and ASD-specific models of anxiety which can then guide treatment for anxiety more effectively. 


\section{Acknowledgement}

We thank Professor Patricia Howlin and Dr Tim Williams for their comments on the manuscript and assistance in development of the study, and Ms Charlotte Hibberd for her invaluable help with the transcript. We are grateful to all parents and children who participated in the focus groups for their time and valuable insights.

\section{Funding}

This research received no specific grant from any funding agency in the public, commercial or non-for-profit sectors.

\section{References}

American Psychiatric Association (2000) Diagnostic and Statistical Manual of Mental Disorder, $4^{\text {th }}$ Edition, Text Revision (DSM-IV-TR). Washington, DC: American Psychiatric Association.

Aronson, J. (1994) A Pragmatic View of Thematic Analysis. The Qualitative

Report, 2. On line journal: http://www.nova.edu/ssss/QR/Backlssues/QR21/aronson.html

Beck, A. T., and Clark, D. A. (1997) An information processing model of anxiety: Automatic and strategic processes. Behaviour Research and Therapy, 35, 49-58. 
Beesdo, K., Knapp, S. and Pine, D. (2009) Anxiety and anxiety disorders in Children and Adolescents: Developmental issues and implications for DSM-V. Psychiatric Clinics of North America. 32, 483-524.

Bellini, S. (2004) Social skills deficits and anxiety in high functioning adolescents with autism spectrum disorders. Focus on Autism and Other Developmental Disabilities, 19, 78-86.

Burnette, C., Mundy, P., Meyer, J., Sutton, S., Vaughn, A., and Charak, D. (2005) Weak central coherence and its relations to theory of mind and anxiety in autism. Journal of Autism and Developmental Disorders, 35, 63-73.

Cartwright-Hatton, S., McNicol, K., and Doubleday, E. (2006) Anxiety in a neglected population: Prevalence of anxiety disorders in pre-adolescent children. Clinical Psychology Review, 26, 817-833.

Chalfant, A., Rapee, R., and Carroll, L. (2006) Treating anxiety in children with high functioning autism spectrum disorders; A controlled trial. Journal of Autism and Developmental Disorders, 37, 1842-1857.

Connolly S.D., Bernstein G.A. (2007) Work group on quality issues: Practice parameter for the assessment and treatment of children and adolescents with anxiety disorders. Journal of the American Academy of Child and Adolescent Psychiatry, 46:2, 267-283 
Costello, E., Mustillo, S., Erkanli, A., Keeler, G. and Angold, A. (2003). Prevalence and development of psychiatric disorders in childhood and adolescence. Archives of General Psychiatry, 60, 837-844

Ehlers, A., and Clarke, D. (2000) A cognitive model of posttraumatic stress disorder. Behaviour Research and Therapy, 38, 319-345.

Einfeld, S. L., and Tonge, B. J. (1992) Manual for the developmental behaviour checklist. Clayton, Melbourne and Sydney: Monash University Centre for Developmental Psychiatry and School of Psychiatry, University of NSW.

Farrugia. S., and Hudson, J. (2006) Anxiety in adolescents with Asperger syndrome: Negative thoughts, behavioral problems, and life interference. Focus on Autism and Other Developmental Disabilities, 21, 25-35.

Fern, E.F. (2001). Advanced Focus Group Research. Thousand Oaks, Calif: Sage.

Gaus, V. L. (2007) Cognitive-Behavioural Therapy for Adult Asperger Syndrome. New York: Guilford press.

Gillott, A., Furniss, F., and Walter, A. (2001) Anxiety in high-functioning children with autism. Autism, 5, 277-286. 
Green, J., Gilchrist, A., Burton, D., and Cox, A. (2000) Social and psychiatric functioning in adolescents with asperger syndrome compared with conduct disorder. Journal of Autism and Developmental Disorders, 30, 279-293.

Green, S.A., and Ben-Sasson, A. (2010). Anxiety disorders and sensory overresponsivity in children with autism spectrum disorders: Is there a causal relationship? Journal of Autism and Developmental Disorders, 40, 1495-1504.

Grudens-Schuck, N.B., Allen, L., and Larson, K. (2004). Focus Group Fundamentals. Ames, lowa: lowa State University Extension. Retrieved on $18^{\text {th }}$ April 2011 at http://www.extension.iastate.edu/publications/pm1969b.pdf Jahoda, A., Selkirk, M., Trower, P., Pert, C., Stenfert, K. B., Dagnan, D., and Burford, B. (2009) The balance of power in therapeutic interactions with individuals who have intellectual disabilities. British Journal of Clinical Psychology, 48, 63-77.

Kitzinger, K. (1995). Introducing focus groups. British Medical Journal, 311, 299-302.

Krueger, R., and Casey, M.A. (2000). Focus Groups: A Practical Guide for Applied Research ( $3^{\text {rd }}$ Edition). Thousand Oaks, Calif: Sage.

Lang, R., Regester, A., Lauderdale, S., Ashbaugh, K., and Haring, A. (2010). Treatment of anxiety in autism spectrum disorders using cognitive behaviour therapy: a systematic review. Developmental Neurorehabilitation, 13(1), 5363. 
MacNeil, B. M., Lopes, V. A., and Minnes, P. M. (2009) Anxiety in children and adolescents with autism spectrum disorders. Research in Autism Spectrum Disorders, 3, 1-21.

Moree, B., and Davis, T.E. (2010) Cognitive-behaviour therapy for anxiety in children diagnosed with autism spectrum disorders: Modification trends. Research in Autism Spectrum Disorders, 4, 346-354.

Muris, P. and Broeren, S. Twenty-five years of research on childhood anxiety disorders: Publication trends between 1982 and 2006 and a selective review of the literature. Journal of Child and Family Studies, 18, 388-395

Muris, P., Steerneman, P., Merckelbach, H., Holdrinet, I., and Meesters, C. (1998) Comorbid anxiety symptoms in children with pervasive cevelopmental cisorders. Journal of Anxiety Disorders, 12, 387-393.

Rapee, R.M, Schniering, C.A., and Hudson, J.L. (2009) Anxiety disorders during childhood and adolescence: Origins and treatment. Annual Review of Clinical Psychology, 5:3, 11-41.

Schniering, C., Hudson, J. and Rapee, R. (2000) Issues in the diagnosis and assessment of anxiety disorders in children and adolescents. Clinical Psychology Review, 20, 453-478.

Simonoff, E., Pickles, A., Charman, T., Chandler, S., Loucas, T., and Baird, G. (2008) Psychiatric disorders in children with autism spectrum disorders: prevalence, comorbidity, and associated factors in a population-derived 
sample. Journal of the American Academy of Child and Adolescent Psychiatry, 47, 921-929.

Stewart, M. E., Barnard, L., Pearson, J., Hasan, R., and O'Brien, G. (2006) Presentation of depression in autism and Asperger syndrome. A review. Autism, 10, 103-116.

Sze, K. M., and Wood, J. J. (2008) Enhancing CBT for the treatment of autism spectrum disorders and concurrent anxiety. Behavioural and Cognitive Psychotherapy, 36, 403-409.

White, S., Oswald, D., Ollendick, T., and Scahill, L. (2009) Anxiety in children and adolescents with autism spectrum disorders. Clinical Psychology Review, 29, 216-229.

Wood, J., Drahota, A., Sze, K., Har, K., Chiu, A., and Langer, D. (2009) Cognitive behavioral therapy for anxiety in children with autism spectrum disorders: A randomized, controlled trial. Journal of Child Psychology and Psychiatry, 50, 224-234.

Wood, J.J., and Gadow, J.D. (2010). Exploring the nature and function of anxiety in youth with autism spectrum disorders. Clinical Psychology: Science and Practice, 17(4), 281-292. 


\section{Appendix: Topic Guide}

1) When does your child become anxious?

Prompts: what are the triggers/ what situations are associated with anxiety?

2) How do you know your child is anxious?

i) What are the behavioural signs that your child is anxious? Prompts: how do they show their anxiety, what do they do?

ii) Do they report any thoughts associated with their anxiety? Prompts: What are the main themes of the negative thoughts? Do they report any positive, coping thoughts? How easy do you think they find it to access their thoughts? Do they report any images?

iii) Does your child have somatic problems associated with their anxiety?

3) How would someone who didn't know your child know they were anxious? Prompts: What would they think or understand about what was happening?

4) What is the difference between children with autism who are anxious and typically developing children who are anxious? 